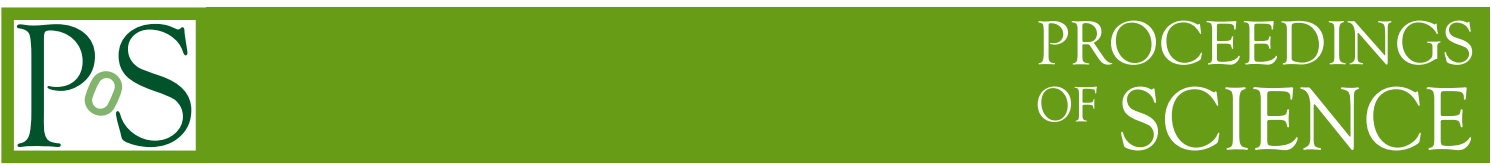

\title{
Supernovae type la: non-standard candles of the Universe
}

\section{A. I. Bogomazov}

Sternberg Astronomical Institute, Lomonosov Moscow State University

E-mail: bogomazov@sai.msu.ru

\section{A. V. Tutukov}

Institute of astronomy, Russian Academy of Sciences

E-mail: atutukov@inasan.ru

\begin{abstract}
We analyze the influence of the evolution of light absorbtion by grey dust in SNe Ia host galaxies and the influence of the evolution of average total mass of coalescing double carbon-oxygen white dwarfs (progenitors of SNe Ia) under the influence of gravitational radiation on the interpretation of Hubble diagrams of SNe Ia. Significant increase in the average energy of SNe Ia due to increase in the total mass of merging dwarfs can be observed at red shift $z>2$. The observed dependence of the distance modulus from the red shift in observations of SNe Ia can be explained not only by the assumption about accelerated expansion of the Universe, but also by the evolution of the absorbtion of light by grey dust in various types of host galaxies of SNe Ia, by the effects of observational selection and by the decrease in the average mass of coalescing degenerate dwarfs.
\end{abstract}

25th Texas Symposium on Relativistic Astrophysics - TEXAS 2010

December 06-10, 2010

Heidelberg, Germany 
In the paper $[1]^{1}$ we showed that the average total mass of double carbon-oxygen $(\mathrm{CO})$ white dwarfs (WD) coalescing under the influence of gravitational waves should evolve with time. Also we calculated the frequency of mergings of $\mathrm{CO}$ WDs and constructed mass distribution of merging CO WDs. See Figure 1 for the mass distribution of CO WDs and Figure 2 for the evolution of average total mass. Such studies are important, because at the end of the last century two groups of SNe Ia observers concluded that the Universe expands with acceleration [3, 4]. Consequently, the understanding of physics SN Ia (which determines the possible evolution of their brightness over time) is necessary to understand the fundamental properties of the Universe. Also we consider here the influence of the evolution of the absorbtion of light by the grey dust in host galaxies of SNe Ia. The absorbtion of light by such dust can lead to the same visible effect of darkening of distant $\mathrm{SNe}$ Ia as expected from the accelerated expansion of the Universe.

Currently, the most popular explanation for SN Ia is mergers of two carbon-oxygen white dwarfs (CO WDs) under the action of gravitational radiation, provided the total mass of the merging dwarfs exceeds the Chandrasekhar limit. This scenario for the formation of SN Ia was suggested in the early 1980s [5, 6]. Apart from mergers of two CO dwarfs, possible origins include the thermonuclear explosion of a WD in a semi-detached system during the accretion of matter from its companion, when the dwarf's mass exceeds the Chandrasekhar limit, and a thermonuclear explosion of a white dwarf with a helium donor star companion; under certain conditions, the mass of the WD may be lower than the Chandrasekhar limit (see, for example, [7]). Here, however, we consider the basic scenario for the formation of SN Ia to be the merging of two CO dwarfs whose total mass exceeds the Chandrasekhar limit [8].

Brightness of SNe Ia can be weakened by the absorbtion in the material around the precursor of a supernova, as well as by the absorbtion by the dust in the host galaxy of the supernova. The most important characteristics of the dust in the host galaxies of supernovae can change over time: the total mass of the dust, distribution of the dust in the galaxy and the composition of the dust. Absorbtion by the interstellar grey dust can lead to overestimated photometric distances in comparison with the universe without the dust. Absorbtion by the intergalactic grey dust was suggested as an explanation of distant SNe Ia darkening instead the acceleration of the expansion of the Universe in papers [9] just after classical works [3, 4].

In our work we study the absorbtion of light by interstellar dust. To estimate the absorbtion of light of SNe Ia in disk galaxies we use a model of the evolution of galaxies from papers [10][12]. The mass of the simulated galaxy was taken to be equal to $2 \cdot 10^{11} M_{\odot}$, and the radius $\sim 10^{4}$ pc. Star formation rate is estimated from the condition of complete ionization of the gaseous disk of the galaxy by the young massive stars. The thickness of the gaseous disk is estimated from the condition of equality of the energy of the SNe explosions converted into the turbulent motion of the gas in the galaxy and by the energy dissipation rate of the turbulent motion of the gas. Frequency of SNe explosions determines the star formation rate in the simulated galaxy, and the dissipation turbulent motion of the interstellar gas is determined by the collisions of the interstellar gas clouds between each other. At the same time it is essential that extinction in elliptical galaxies is negligible in comparison to disk galaxies.

\footnotetext{
${ }^{1}$ In this paper we presented the results of population synthesis made using the "Scenario Machine" (computer code designed for studies of the evolution of close binary stars, a description of the code can be found in [2].
} 


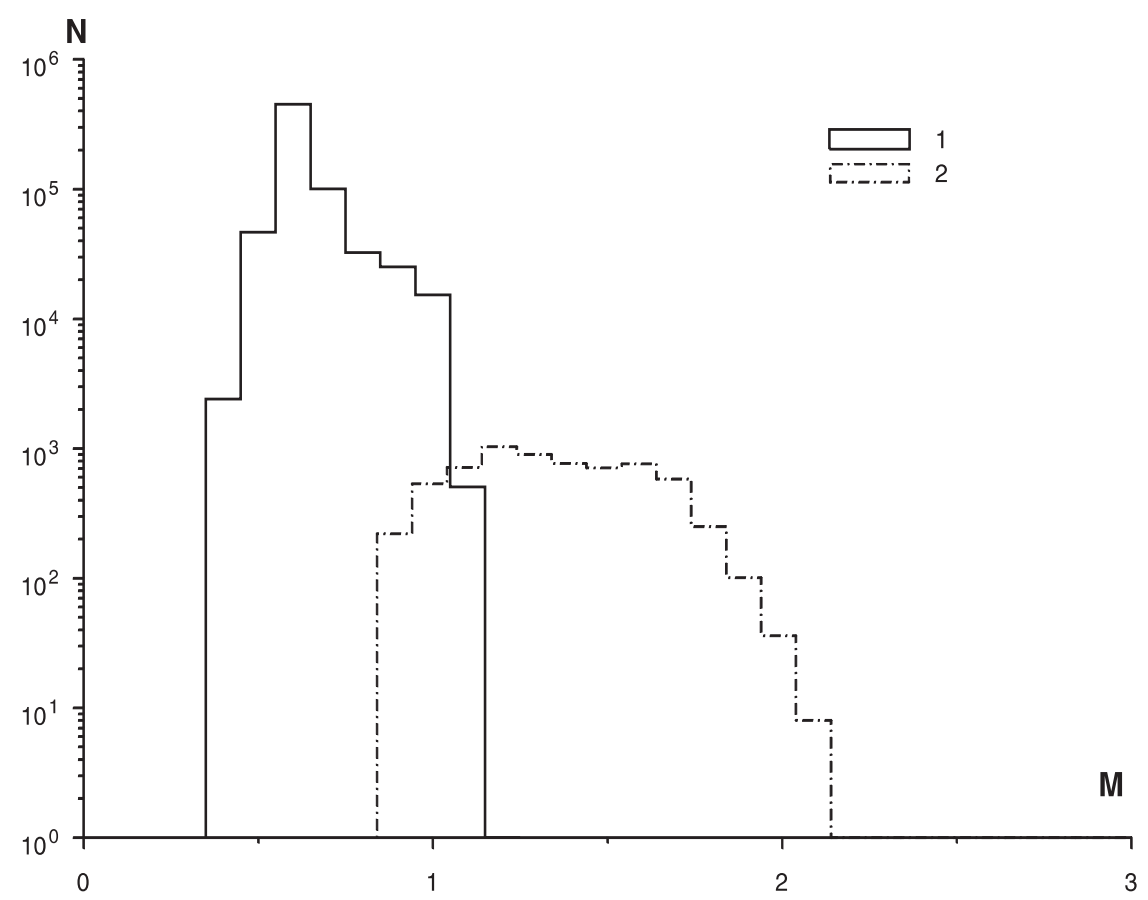

Figure 1: The mass distribution for carbon-oxygen WDs that have not undergone mergers (solid curve) and the total mass distribution for merging WDs (dash-dotted curve). The horizontal axis plots the mass of the WD in $M_{\odot}$ (the total mass of the two WDs in the case of merging WDs), and the vertical axis the number of WDs (and of mergers) derived from the calculations.

Is is important to note that we take into consideration so-called "grey" dust. Light absorbtion by such dust does not depend on the light wavelength. This kind of dust has already been discovered in our own Milky Way Galaxy [13]. Small dust particles can be taken into account due to the light reddening at least in principle. Also it is important to take into account that modern simulations of the evolution of the optical depth of the galaxy is not completely defined and it is not possible to estimate the optical depth with precision better than by a factor of $2[10,11]$. Light absorbtion by the grey dust does not depend on the SNe Ia model. We suppose that the abundance of the grey dust in the host galaxies of $\mathrm{SNe}$ Ia is proportional to the abundance of all kinds of dust in these galaxies.

We assume that the average absolute magnitude of type Ia supernovae depends on the average total mass of merging CO WDs as follows: $M=C-2.5 \cdot \log M_{\Sigma}$, here $M$ is the average absolute magnitude of SNe Ia, $M_{\Sigma}$ is the average total mass of merging CO WDs, $C$ is a constant value. In Figures 3 and 4 we present the dependence of value $\Delta(m-M)$ from the red shift $z: \Delta(m-M)=$ $\left(m_{1}-C\right)-\left(m_{2}-C\right)=m_{1}-m_{2}$, here $m$ and $M$ are visible and absolute magnitudes correspondingly, $m_{1}$ is the visible magnitude, evolution of the average total mass of merging CO WDs is taken into account, $m_{2}$ is the visible magnitude calculated in supposition that SNe Ia are standard candles with absolute magnitude $C$.

According to our calculations (see Figures 3 and 4) the average energy of SNe Ia should increase with red shift at $z>2$ and such increase should become significant at red shift $z \gtrsim 8$, because the average total mass of merging dwarfs was greater during early stages of evolution of 


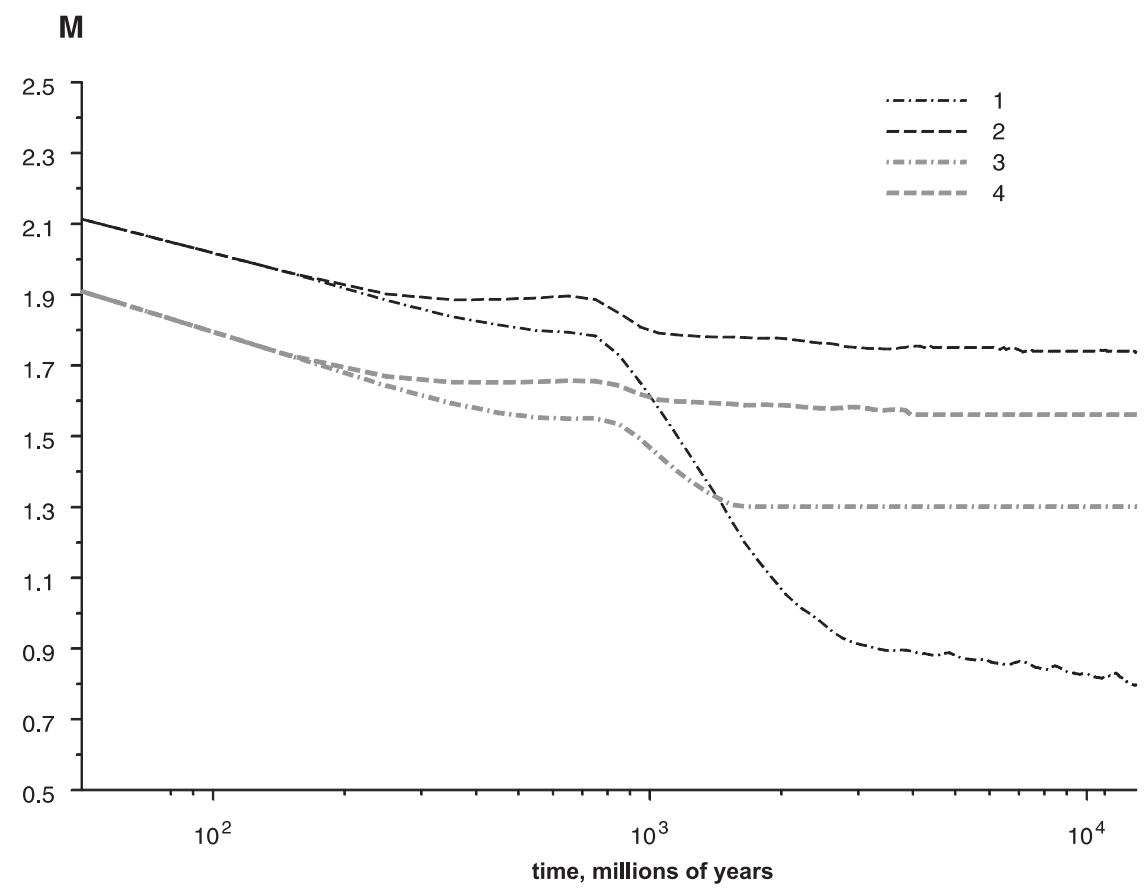

Figure 2: The average mass of merging WDs as a function of time from the onset of the star formation (in solar masses). The numbers at the top mark the curves corresponding to mergers of 1 all WDs, 2 WDs of all possible chemical compositions, but taking into account only mergers in which the total mass of the WDs exceeds the Chandrasekhar limit, 3 carbon-oxygen WDs, and 4 carbon-oxygen WDs with the total mass exceeding the Chandrasekhar limit. The star formation history similar was taken from [14].

the Universe. There are no known supernovae at so high red shift. Therefore this conclusion can not affect the result of classical papers [3,4]. At the same it is important to take into account that in our population synthesis models we study the evolution of a population of stellar objects (any galaxy), which was born at a definite moment of time (at red shift $z^{*}=10$ in this work), then this population evolves. If there is a significant burst of star formation after $z^{*}$ we should compare our results with the period of time elapsed from this star formation burst. Also independently from the population age a dispersion of SNe Ia parameters should be due to the fact that total mass of merging dwarfs can vary approximately by a factor of 1.5 (see Figure 1).

The evolution of the absorbtion of light by the interstellar dust according to the model under consideration in this work can lead to the much higher decrease of brightness of distant $\mathrm{SNe}$ in comparison with the assumption about the dark energy existence in the Universe. At the same time the curve 4 in Figures 3 and 4 describes the absorbtion by all kinds of dust. If we change internal parameters of the dust evolution model and set up required abundance of grey dust we can make (in principle) practically ideal coincidence between curves 2 and 4 in a very wide range of red shift. Nevertheless such method is under critique, because fine tuning of the model parameters is an unattractive way to explain physics [15].

It was shown that $\mathrm{SNe}$ Ia are $\approx 0^{m} .1$ brighter in quiet galaxies than in galaxies with active star formation [16]. The dependence between the SNe Ia brightness and the type of SNe Ia host galaxies also was mentioned in the paper [17]. 


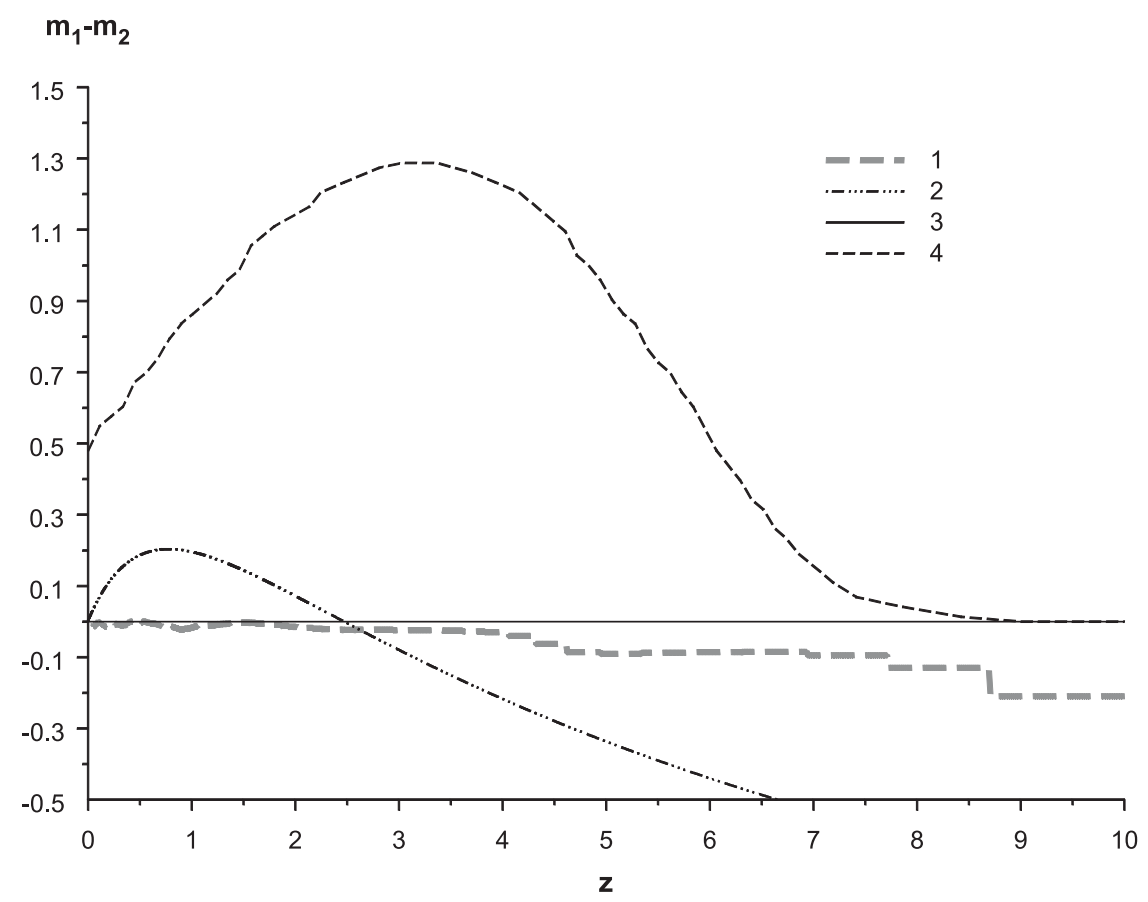

Figure 3: In this figure we plot the difference $\Delta(m-M)$ between dependencies of the SNe Ia magnitude on the red shift of SNe Ia with the effects under consideration (we consider the evolution of absorbtion of light by dust and the evolution of average total mass of merging CO WD) and the same dependencies calculated under the assumption that SNe Ia are standard candles and the absorbtion of light by dust does not evolve. The curve 1 is calculated under the assumption that there is no absorbtion and the absolute magnitude of $\mathrm{SNe}$ Ia evolves from its maximum value in the beginning of the evolution to a constant value approximately after one billion years of evolution of stars in galaxies, it corresponds to the curve 4 in fig. 2, we use the next set of cosmological parameters for this curve: $\Omega_{\lambda}=0, \Omega_{M}=0.2, \Omega_{t o t}=0.2, H_{0}=70 \mathrm{~km} \mathrm{~s}^{-1} \mathrm{mpc}^{-1}$, $z^{*}=10$. The curve 2 is calculated under the assumption that absorbtion does not play any role, the absolute stellar magnitude of SNe Ia does not evolve and $\Omega_{\lambda}=0.7, \Omega_{M}=0.3, \Omega_{t o t}=1, H_{0}=70 \mathrm{~km} \mathrm{~s}^{-1} \mathrm{mpc}^{-1}$. The curve 3 corresponds to the model without evolutionary effects and $\Omega_{\lambda}=0, \Omega_{M}=0.2, \Omega_{\text {tot }}=0.2, H_{0}=70$ $\mathrm{km} \mathrm{s}^{-1} \mathrm{mpc}^{-1}$. The curve 4 describes evolution of absorbtion of light by dust, this dependence was taken from [12] (dependence of absorbtion $A_{v}$ on red shift $z$, mass-radius relation is $M \sim R^{2}$ ).

We offer the following interpretation of our results. As we can see from the figure 3, the growth of the dust absorbtion in disk galaxies takes place at almost the same rate as the additional darkening of the supernovae light grows with the redshift due to the assumption of accelerated expansion of the Universe. Consequently, it is possible to hypothesize that the accelerated darkening of the supernova (in average, which is important) is the result of the evolution of the absorbtion of radiation by the grey dust, the effect of which is not taken into account yet. However, with further increase of the red shift the absorbtion also increases. This means that $\mathrm{SNe}$ fade more and more in comparison with the model without dust. There are a lot of distant supernovae, which belong to the slow down curve rather than to the curve of accelerated expansion of Universe (see, for example, figure 10 in paper [18]). This fact is an argument against the explanation of the accelerated darkening of SNe Ia by the evolution of the absorbtion of light by grey dust. But this argument is valid only if all host galaxies of SNe Ia are disk galaxies with active star formation. This is not so: 


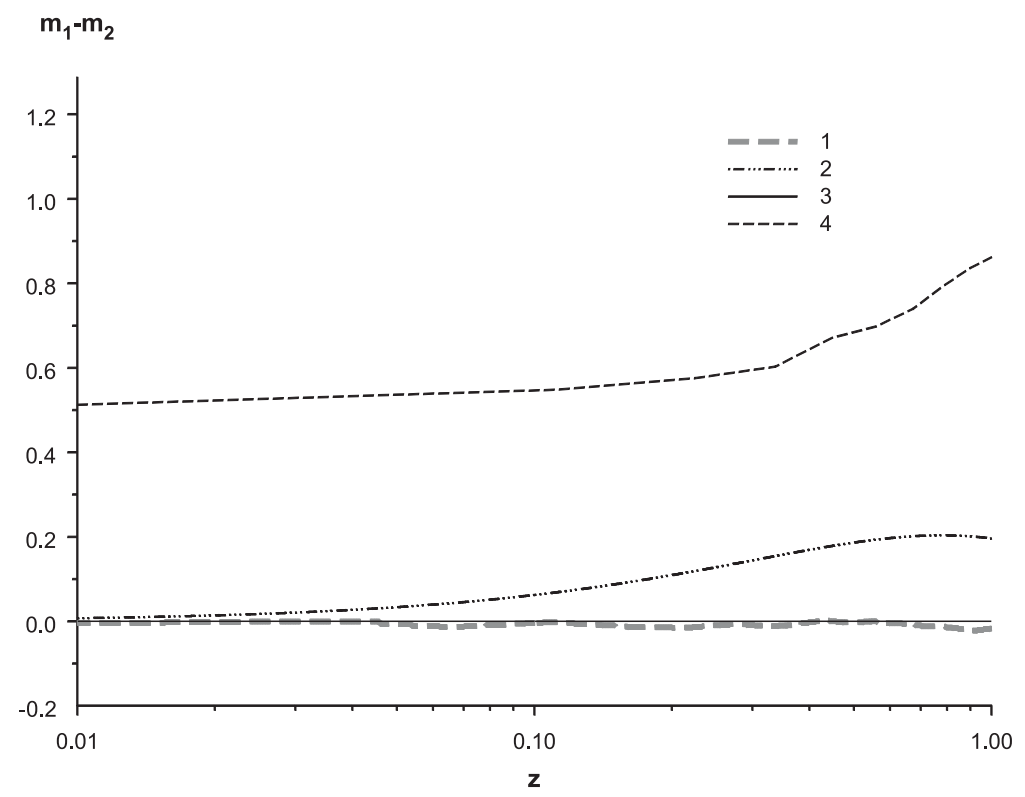

Figure 4: The same as Figure 3 in the red shift interval $z=0.01 \div 1$.

for example, host galaxy of SN $1997 \mathrm{ff}$ (this is one of the most distant SNe Ia) is a quiet elliptical galaxy [19].

As we already mentioned, the absorbtion in elliptical galaxies in comparison with disk galaxies is negligible and it does not evolve during long period of time comparable with Hubble time. We also would like to note that according to population synthesis results (see, for example, figure 1 in paper [20]) the frequency of SNe Ia in an elliptical galaxy drops approximately by a factor of two between 1 and 10 billions of years from the birth of the galaxy. This means that in past the probability of SN Ia explosion is higher in an elliptical galaxy than in a disk galaxy. This fact probably explains observed absence of "accelerated" SNe Ia with red shift $z>1$.

Thus, the use of SNe Ia as cosmological standard candles must take into account not only characteristics of the model of supernovae, but also the evolution of the absorbtion by dust (including the grey dust) in the host galaxies of SNe Ia. It is better to use SNe Ia in galaxies without star formation as distance indicators. SNe Ia in galaxies with active star formation can be used as a probe to study the evolution of the absorbtion in these galaxies.

\section{References}

[1] A. I. Bogomazov, A. V. Tutukov, Merging of components in close binaries: Type Ia supernovae, massive white dwarfs, and Ap stars, Astronomy Reports 53 (2009) 214.

[2] V. M. Lipunov, K. A. Postnov, M. E. Prokhorov, A. I. Bogomazov, Description of the "Scenario Machine”, Astronomy Reports 53 (2009) 915.

[3] A. G. Riess, A. V. Filippenko, P. Challis, et al., Observational Evidence from Supernovae for an Accelerating Universe and a Cosmological Constant, Astronomical Journal 116 (1998) 1009.

[4] S. Perlmutter, G. Aldering, G. Goldhaber, et al., Measurements of Omega and Lambda from 42 High-Redshift Supernovae, Astrophysical Journal 517 (1999) 565. 
[5] A. V. Tutukov, L. R. Yungelson, Evolutionary Scenario for Close Binary Systems of Low and Moderate Masses, Nauchnye Informacii Astron. Sov. Akad. Nauk SSSR 49 (1981) 3.

[6] I. Jr. Iben, A. V. Tutukov, Supernovae of type I as end products of the evolution of binaries with components of moderate initial mass (M not greater than about 9 solar masses), Astrophysical Journal Suppl. Ser. 54 (1984) 335.

[7] A. Ruiter, K. Belczynski, S. Sim, et al., Type Ia Supernova Delay Times and Rates for Double-detonation Sub-Chandrasekhar Mass Models [arXiv:1011.1407].

[8] L. R. Yungelson, Evolution of the number of accreting white dwarfs with shell nuclear burning and the SNe Ia rate, Astronomy Letters 36 (2010) 780.

[9] A. Aguirre, Dust versus Cosmic Acceleration, The Astrophysical Journal 512 (1999) L19.

[10] C. Firmani, A. Tutukov, Evolutionary models for disk galaxies, Astronomy and Astrophysics 264 (1992) 37.

[11] D. Z. Wiebe, A. V. Tutukov, B. M. Shustov, On the evolution of the star formation rate in disk galaxies, Astronomy Reports 42 (1998) 1.

[12] A. Kabanov, A. V. Tutukov, B. M. Shustov, Evolution of star formation rate and extinction in disk galaxies at high red shift, Astronomy Reports 55 (2011) in press.

[13] E. Gorbikov, N. Brosch, Grey Milky Way extinction from SDSS stellar photometry, Monthly Notices of the Royal Astronomical Society 401 (2010) 231.

[14] E. P. Kurbatov, A. V. Tutukov, and B. M. Shustov, Evolution of Galaxies and the Tully-Fisher Relation, Astronomy Reports 49 (2005) 510.

[15] A. G. Riess, L.-G. Strogler, S. Casertano, et al., New Hubble Space Telescope Discoveries of Type Ia Supernovae at $z>=1$ : Narrowing Constraints on the Early Behavior of Dark Energy, Astrophysical Journal 659 (2007) 98.

[16] H. Lampeitl, M. Smith, R. Nichol, et al., The Effect of Host Galaxies on Type Ia Supernovae in the SDSS-II Supernova Survey, Astrophysical Journal 722 (2010) 566.

[17] M. Sullivan, A. Conley, D. A. Howell, et al., The dependence of Type Ia Supernovae luminosities on their host galaxies, Monthly Notices of the Royal Astronomical Society 406 (2010) 782.

[18] M. Kowalski, D. Rubin, G. Aldering, et al., Improved Cosmological Constraints from New, Old, and Combined Supernova Data Sets, Astrophysical Journal 686 (2008) 749.

[19] A. G. Riess, P. Nugent, R. Gilliland, et al., The Farthest Known Supernova: Support for an Accelerating Universe and a Glimpse of the Epoch of Deceleration, Astrophysical Journal $\mathbf{5 6 0}$ (2001) 49.

[20] H. E. Jorgensen, V. M. Lipunov, I. E. Panchenko, et al., Evolution of Supernova Explosion Rates in the Universe, Astrophysical Journal 486 (1997) 110. 